\title{
Development of large size Micromegas detectors for the upgrade of the ATLAS experiments.
}

\section{Michele Bianco*'}

CERN, Switzerland

E-mail: michele.biancolcern.ch

\begin{abstract}
The luminosity upgrade of the Large Hadron Collider at CERN foresees a luminosity increase by a factor 3 compared to the LHC luminosity design value. To cope with the corresponding rate increase, the Muon System of the ATLAS experiment at CERN needs to be upgraded. In the first station of the high rapidity region, micromegas detectors have been chosen as the main tracking chambers but will, at the same time, also contribute to the trigger. We describe the R\&D efforts that led to the construction of the first $\left(1 \times 2.4 \mathrm{~m}^{2}\right)$ large micromegas detectors at CERN and outline the next steps towards the construction of the $1200 \mathrm{~m}^{2}$ of micromegas detectors for the ATLAS upgrade. The technical solutions, adopted in the construction of the chamber as well as results on the detector performance with cosmic rays are shown.
\end{abstract}

The European Physical Society Conference on High Energy Physics -EPS-HEP2013

18-24 July 2013

Stockholm, Sweden

\footnotetext{
* Speaker.

† on behalf of MAMMA (Muon ATLAS Micromegas Activity) Collaboration.
} 


\section{Introduction}

The Micromegas (Micro-MEsh Gaseaus Detector) MM technology was developed in the middle of the 1990's [1]. It consist of a planar (drift) electrode, a gas gap of a few millimetres thickness acting as conversion and drift region, and a thin metallic mesh at typically 100-150 $\mu \mathrm{m}$ distance from the readout electrode, delimiting the amplification region. In the beginning, the drift electrode and the amplification mesh were at negative high voltage (HV) potentials and the readout electrode was at ground potential. The MM operation is based on the ionization of the gas by charged particles traversing the drift space where an electric field of few hundred $\mathrm{V} / \mathrm{cm}$ is applied. The electrons, produced by the ionization, drift towards the mesh and the amplification region, where the signal is created by means of an electric field of about $40-50 \mathrm{kV} / \mathrm{cm}$. The drift of the electrons in the conversion gap depends on the drift gas, the drift distance, and the drift field and it typically takes several tens of nanoseconds, while for the amplification process a fraction of a nanosecond is required. As a conseguence a fast pulse of electrons on the readout strip is produced.

The ions that are produced in the avalanche process, move back to the amplification mesh. The ions evacuation needs about $100 \mathrm{~ns}$, till very fast compared to other detectors.

\section{The Micromegas thecnology for the ATLAS New Small Whell upgrade}

The Large Hadron Collider (LHC) [2] complex will be upgraded in several phases which will allow the physics program to be significantly extended. After the expected long shutdown in 2018 the accelerator luminosity will be increased to $2-3 \times 10^{34} \mathrm{~cm}^{-2} \mathrm{~s}^{-1}$ exceeding the design value of $1 \times 10^{34} \mathrm{~cm}^{-2} \mathrm{~s}^{-1}$ allowing the ATLAS experiment [3] to collect approximately $100 \mathrm{fb}^{-1} /$ year. In order to take advantage of the improved LHC operation the ATLAS detector must be upgraded to have better performance at the expected high luminosity. In particular, the ATLAS muon system will need major improvements in the highest rapidity region. Based on background estimations, a list of requirements for these new detector has been estabilished [ 4 ]:

* Resolution better than $100 \mu \mathrm{m}$ per plane, for each of the two planned 4-layer multi-plane detector that will replace the Small Wheel.

* High single plane detection efficiency $\geq 98 \%$.

* Efficiencies and resolutions should not degrade at very high momenta (due to $\delta$ rays, showers, etc).

* Measure the second coordinate with a resolution of 1-2 mm to facilitate the matching between the MS and the ID track for the combined muon reconstruction.

The highly ionizing particles, produced in the LHC collisions, leads to large energy deposit and an increasing probability for spark occurence [5]. For the MM detectors, to be installed on the New Small Wheel, a spark protection system has been developed adding a layer of resistive strips on top of a thin insulator directly above the readout electrode, in such a way the MM become spark-insensitive [6]. The signals are capacitively coupled to the readout electrode which is not anymore directly exposed to the charge created in the amplification region. 
Another difference for the MM detectors for the NSW from the original MM scheme is the HV distribution configuration. In this scheme, positive HV is applied to the resistive strips while the amplification mesh is connected to ground. In this operation mode, which resulted in a more stable detectors, the spark-induced current can be evacuated very quickly to ground through the mesh, without mesh potential variations. Moreover the better focusing of the field lines between the amplification mesh and the resistive strips leads to a better charge collection on the resistive strip. Finally, in the MM layout for the ATLAS New Small Whell upgrade the amplification mesh is not integrated in the readout structure.

The mesh decoupling from the readout structure, presents several advantages for large-area detectors:

* The mesh size is only limited to he mesh fabrication size and stretching machines and not to the size of the individual PCBs.

* It facilitates detector opening and cleaning.

* It separates PCB production from mechanical construction.

Figure 1 shows schematically the internal structure of a readout PCB. The readout strips are drawn on $0.5 \mathrm{~mm}$ thick PCBs and then covered by a $64 \mu \mathrm{m}$ thick layer of insulator, followed by the resistive strips. On top of the resistive strips the mesh support pillars are deposited. The mesh sits on these support pillars, however it is not a part of the readout PCB but a part of the drift panel.

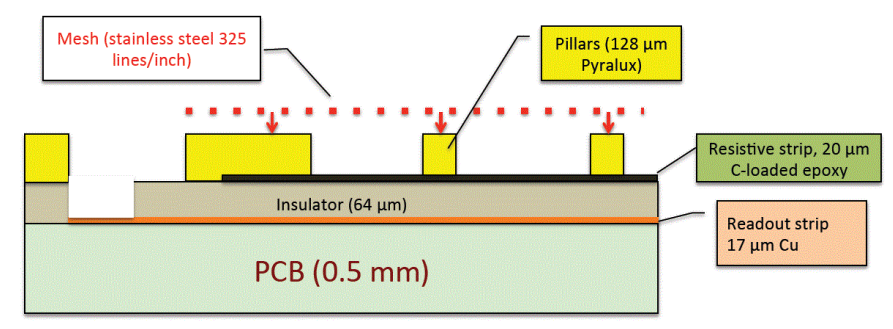

Figure 1: Internal structure of the MM readout boards, in this cinfiguration the mesh is not part of the readout $P C B$

\section{The large MicroMmgas chamber $\left(1 \times 2.4 \mathrm{~m}^{2}\right)$ construction}

The realization of the ATLAS NSW, requires the production of large size MM [7]. In the current layout, the largest MM will have a trapezoidal shape, with a surface of $3 \mathrm{~m}^{2}$. In order to prove the ability to construct a detector of that size with the adequate mechanical precision, a working prototype with the dimensions $\left(1 \times 2.4 \mathrm{~m}^{2}\right)$ has been built at CERN laboratories with the help of the PH-DT-EO unit and the CERN PCB workshop.

With the mesh decoupling from the readout structure,two types of panels are needed to assemble an operational detector, the readout panel, carrying the readout boards, and the drift panel which comprises the drift electrode and the amplification mesh. This is schematically shown in Fig. 2. 

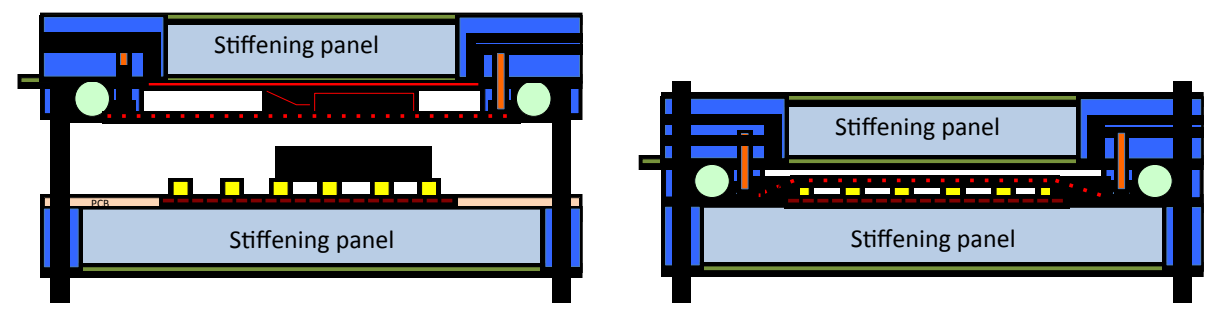

Figure 2: Schematics of a single MM plane assembly showing the drift and readout panels in open (left) and closed (right) position.

Each panel would be composed of a stiffening structure onto wchich either of the electrode structure would be glued. Stiffening panels consist of aluminum bars filled with honeycomb and spacers while the electrodes consist of 8 PCBs ( 4 for the readout plane and 4 for the drift). The PCBs that compose the readout panel host 4096 strips which are read from the short sides of the chamber. The assembly of the panels was done on a granite table equipped with a vacuum sucking system. The vacuum sucking system was made by a thin layer of a mesh fabric covered by a $175 \mu \mathrm{m}$ thick mylar foil. The mylar foil with a grid of small holes, was sealed against the granite table. The PCBs (both in case of the readout electrode that in cas of drift electorde) to be glued to the honeycomb were placed face down on the mylar foils and and aligned, than the gaps between the boards were closed by tape as well the PCB edges to prevent glue from creeping under the boards.

By means of the sucking system the PCBs were retained and fixed on the granite table. The aluminum profiles were glued to the boards using epoxy glue, while an expanding glue was used to glue the honeycomb on the readout boards, the glue take 24 hours to cure completely. The second face of each panel was glued using tha same technique of the sucking system, but in this case, the positioning of the half-panel already built, was controlled by stiff-back tool which kept the panel at a fixed distance. The stiff-back is a tool with a flat lower surface and provisions for vacuum sucking. For this exercise a simplified stiff-back structure was used, it was realized by use of aluminum bars equipped with sucking heads approximately every $25 \mathrm{~cm}$. The bars were precisely machined and glued together with longitudinal bars, stiff enough to keep their structural form after the curing of the glue; thus forming a plane that matches the surface of the granite table.

The main differences between readout and drift panel, involved in the panel production, can be summarized as follow: i) the skins of the drift panels have flat surfaces, while the readout boards have the $128 \mu \mathrm{m}$ high pillars, ii) a solid profile is embeded in the drift panel frame onto the mesh frame will be installed, iii) an open channel that serves as gas manifold is integrated into the drift panel frame, only on the shorter sides.

In correspondence of the solid profile embeded in the drift panel, an aluminum frame was attached were the mesh was glued with a tension of about $10 \mathrm{~N} / \mathrm{cm}$ by specialized company. For the glueing a cyanolit-based glue is used, and the mesh is secured with a second line of epoxy glue. The mesh is also glued to the drift panel spacers, the latter have the same height as the mesh support frame. As last step, before the chamber assembly, three small openings into the mesh at the place of the drift panel spacers were made to create the passages for the panel interconnections. To 
assure the cleanliness during the assembly and avoid dust particles that can produce current bridges over the $128 \mu \mathrm{m}$ distance between the mesh and the readout structure, the final assembly was done in controlled clean room of class 10000. In Fig. 自 the 3D view rendering of the large Micromegas project and the final chamber after the assembling are shown.
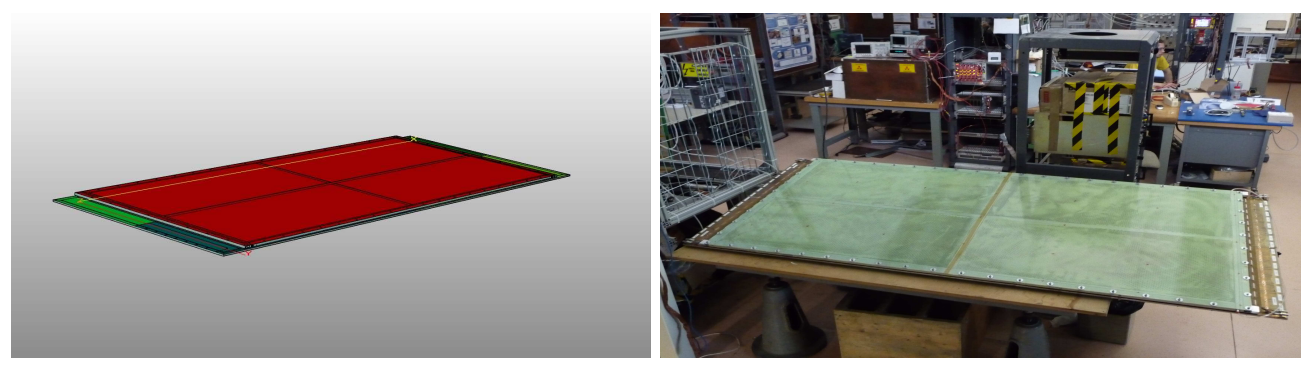

Figure 3: The 3D rendering and a picture of the large Micromegas chamber after the assembling.

\section{First large MicroMegas chamber characterization}

Since no test-beam were available at CERN at the time of the large chamber construction, a dedicated cosmic stand was installed in the RD51 laboratoryt. Three planar scintillators, about $0.25 \times 1.5 \mathrm{~m}^{2}$ large, were positioned above the chamber to provide a cosmic muon trigger. The scintillator configuration with rispect to the chamber, was changed according to the study of interest. The chamber was operated with $\mathrm{Ar} / \mathrm{CO}_{2} 97 / 3 \%$ gas mixture with a flow of about $10 \mathrm{l} / \mathrm{hour}$, the drift plane was set to 300 Volt while the resistive strips were set at 560/580 Volt.

The data acquisition was based on the so-called Scalable Read-out System (SRS) [9] developed at CERN. The detector readout was done through the APV25 readout chip [8] using an adapter (ADC) and FrontEndConcentrator (FEC) cards to digitize the analog data. The FEC board receives the external trigger signal as a NIM or LVDS signal and reads out to a computer via an ethernet link. Both ADC and FEC boards are installed in a standard 6U Eurocrate. Each SRS DAQ unit can read-out 2048 detector channels, same number of strips hosted by each readout side of the large chamber. To provide the cosmic trigger, the three scintillator were aligned along the long chamber side a the center of the chamber. To assess the uniformity of the responce of large chamber, a long runs was recorded, for each chamber readout side.

The studies of the chamber performances are based on the properties of the collected clusters. A cluster is a group of fired strips with charge above a given threshold. Our clusterization algorithm is initiated with a scan over all strips in the detector. A strip is selected if it contains a signal with a pulse-height that passes a predefined threshold. Neighboring selected strips are merged into a cluster, and the clusterization continues also if one strip fails the selection. Our algorithm hence selects clusters with no more than one hole, made by a single strip, and allows for multiple clusters per event.

In Fig. \#, for one of the readout side, the number of cluster versus the strip position is shown for all events and for events with only one cluster. The obtained profile comes from the trigger coverage convoluted with the cosmic rays angular distribution $\left(\propto \cos ^{2} \Theta\right)$, indeed the events fall mostly in 
the center of the chamber. The trigger coverage became more evident when the events with only one cluster are selected.

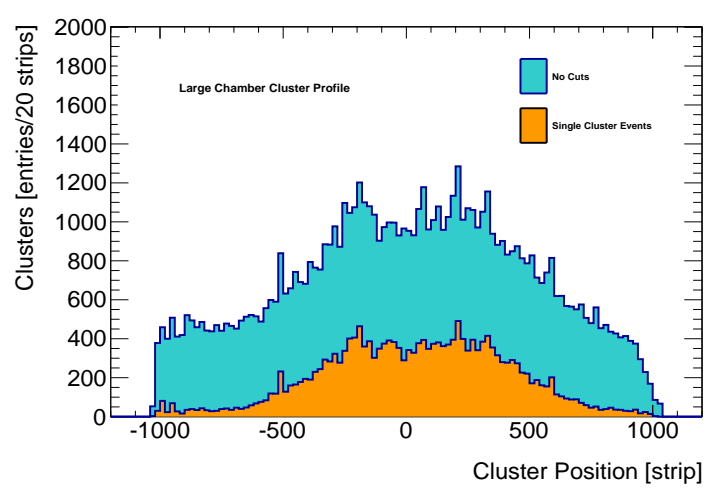

Figure 4: Number of clusters vs strip position for one side of the large chamber under test.

In order to analize the chamber responce along the strip lengh a surface scan was performed. The trigger configuration was modified, the scintillator closest to the chamber was turned by $90^{\circ}$. By moving the turned scintillator, events impinging in a specific part of the detector were selected, the whole detector was tested in different steps. At each step, the chamber response through the cluster distribution, the cluster charge as well as the shape of the acquired signals and their rise time were studied. A uniform behaviour in spatial, charge and time responce was observed along the whole strip leght. In Fig. 5 the Micromegas cluster distribution and cluster charge versus strip position are presented, while in Fig. 6 the signal shape for different steps, as a function of the distance from the front end are shown.
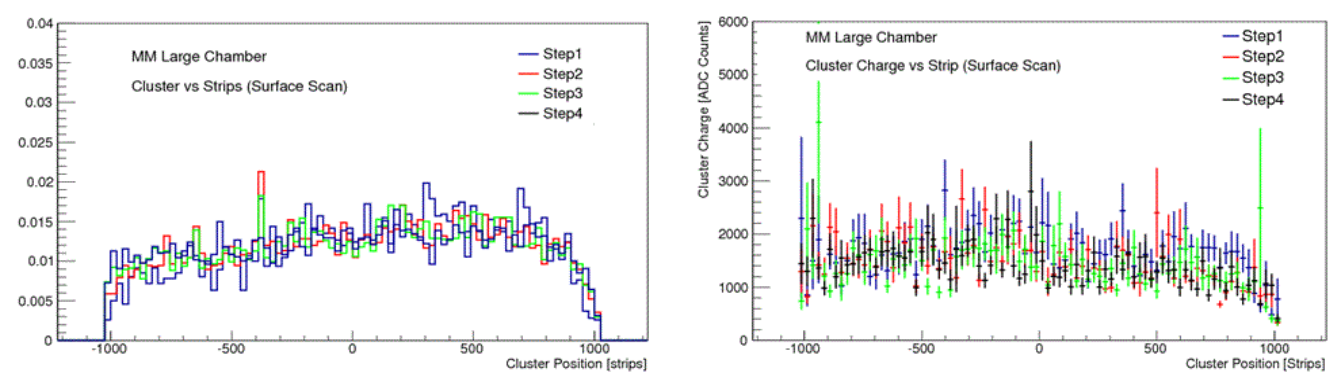

Figure 5: Number of cluster and cluster charge vs strip position as a function of the distance from the front end.

\section{Conclusion}

The construction and assembly of the largest Micromegas chamber ever built and its first chamber characterization with cosmics were presented. The chamber built was smoothly operated and shows a reasonably uniform responce over the full detector area. With the performed surface scan no signal reduction was observed over the $1 \mathrm{~m}$ strip lengh. The Micromegs chamber thecnology here presented is fully suitable for the upgrade of the ATLAS NSW. 

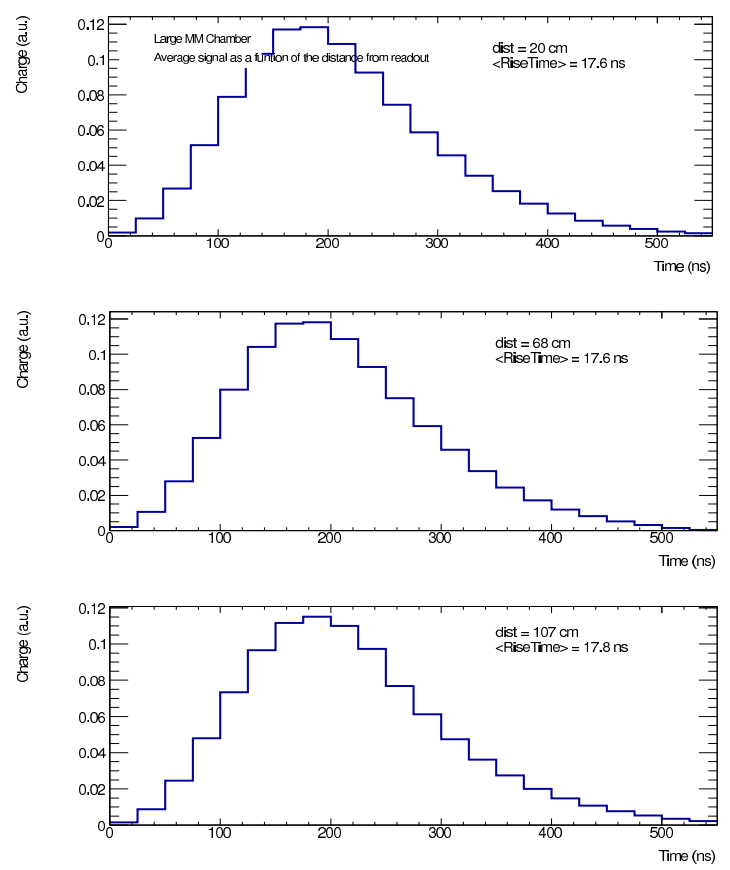

Figure 6: Signal shape for three differents distance from the front end.

\section{References}

[1] Y. Giomataris, P. Rebourgeard, J.P. Robert and G.Charpak, Micro-Pattern Gaseaus Detectors, Nucl. Instrum. and Meth. A376 (1996) 29-35.

[2] LHC Collaboration, The CERN Large Hadron Collider : Accelerator and Experiments, JINST 3 (2008) S08001

[3] ATLAS Collaboration, The ATLAS Experiment at the CERN Large Hadron Collider, JINST 3 (2008) S08003.

[4] ATLAS Collaboration, ATLAS New Small Wheel TDR, CERN-LHCC-2013-006 ATLAS-TDR-020-2013.

[5] H. Raether, Z. Phys 112 (1939) 464.

[6] T. Alexopoulos, et al.,A spark-resistant bulk-micromegas chamber for high-rate applications, Nucl.Instrum.Meth. A640 (2011) 110-118

[7] T.Alexopoulos, et al., Development of large size Micromegas detector for the upgrade of the ATLAS muon system, Nucl.Instrum.Meth. A617 (2010) 161-165

[8] L. Jones, APV25-S1: User guide version 2.2, RAL Microelectronics Design Group (2001), pg. 20, http://hep.ucsb.edu/people/affolder/User Guide 2.2.pdf.

[9] Scalable Readout System, https://espace.cern.ch/rd51-wg5/srs/default.aspx. 